\title{
Mediastinitis necrotizante descendente de origen odontogénico
}

\author{
Alfonso Muñoz R. ${ }^{1}$, Edgard Riquelme M. ${ }^{1}$, Víctor Sanhueza O. ${ }^{1}$ y Daniel Reyes C. ${ }^{1}$
}

\section{Descending necrotizing mediastinitis of odontogenic origin}

Introduction: Inappropriate and ineffective treatment of odontogenic infections can cause life-threatening complications such as Descending Necrotizing Mediastinitis (MND). MDN is a serious infection that affects the neck-thorax, with a high mortality rate from sepsis and organ failure if it is not treated quickly and effectively. Aim: To describe a case of MND of odontogenic origin and its medical-surgical management. Case report: We present a 34-year-old male patient who is admitted with an acute infectious condition of odontogenic origin, which compromises spaces of the head, neck and thorax (upper mediastinum), which is treated successfully. Discussion: Odontogenic infections are generally localized and can be treated by conventional therapies. In spite of this, if these infections cannot be controlled, either by not carrying out appropriate treatments or by immunosuppressed states of the patient, different complications such as MND can develop. Conclusion: A rapid diagnosis, aggressive surgical treatment, adequate antibiotic therapy and supportive care are the fundamental pillars for the management of MND.

Key words: complication; mediastinitis; descending necrotizing mediastinitis; odontogenic infection; transcervical drainage.

\section{Resumen}

Introducción: El tratamiento inoportuno e ineficaz de las infecciones odontogénicas puede causar complicaciones potencialmente mortales como la mediastinitis necrotizante descendente (MND). La MDN es una infección grave que afecta al cuello-tórax, con una alta tasa de mortalidad por sepsis e insuficiencia orgánica si no se trata de manera rápida y efectiva. Objetivo: Describir un caso de MND de origen odontogénico y su manejo médico-quirúrgico. Caso clínico: Presentamos un paciente de sexo masculino de 34 años que ingresa con un cuadro infeccioso agudo de origen odontogénico, que compromete espacios de la cabeza, cuello y tórax (mediastino superior), el cual se trata exitosamente. Discusión: Las infecciones odontogénicas son generalmente localizadas y que se pueden tratar mediante terapias convencionales. A pesar de esto, si estas infecciones no pueden controlarse, ya sea por no realización de tratamientos oportunos o por estados inmunosuprimidos del paciente, se pueden desarrollar diferentes complicaciones como la MND. Conclusión: Un diagnóstico rápido, el tratamiento quirúrgico agresivo, la terapia antibiótica adecuada y la atención de apoyo son los pilares fundamentales para el manejo de la MND.

Palabras clave: complicación; mediastinitis; mediastinitis necrotizante descendente; infección odontogénica; drenaje transcervical.
'Hospital de Urgencia Asistencia Pública Dr. Alejandro del Río. Santiago, Chile.

Recibido 2019-07-11 y aceptado 2020-01-20

Correspondencia a: Dr. Daniel Reyes C. reyescourt@yahoo.es

\section{Introducción}

La mediastinitis necrotizante descendente (MND) es una infección polimicrobiana aguda y rápidamente progresiva de los tejidos conectivos mediastínicos y de las estructuras que los rodean; que parte de una infección severa de origen odontogénico, faríngea o cervical que se propaga por los espacios cervicales profundos hacia la cavidad torácica, causando necrosis tisular con altas tasas de mortalidad (10\%$40 \%$ ) por sepsis y falla orgánica si no se trata de manera rápida y adecuada ${ }^{1,2}$.

En 1938, Pearse describió por primera vez un caso de mediastinitis resultante de una infección orofaríngea ${ }^{3}$. De los casos reportados de MND un $60 \%$ a $70 \%$ son de origen odontogénico, particularmente cuando el segundo o tercer molar mandibular está involucrado ${ }^{4}$. Adicionalmente, la incidencia de 
MND como complicación a exodoncia de terceros molares impactados o cirugía de implantes dentales, ha ido en aumento gradualmente ${ }^{5}$.

La vía de diseminación de la infección es por compromiso de los espacios del cuello, partiendo en la región orofaríngea o submandibular, el espacio parafaríngeo limitado hacia medial por la fascia bucofaríngea, y el músculo constrictor superior de la faringe y lateralmente por la rama mandibular y los músculos pterigoideos. Del espacio parafaríngeo se difunde al lado contralateral a través de la pared parafaríngea posterior y desciende hacia el espacio pretraqueal por anterior y al espacio prevertebral por posterior. Otra vía de diseminación es desde el espacio submandibular o pterigomandibular a través de la vaina carotídea hasta el mediastino y espacio pleural posterior. Este descenso es facilitado por la gravedad, respiración y la presión torácica negativa ${ }^{6}$.

La MND tiene un curso fulminante con riesgos potenciales de sepsis, piotórax, pericarditis, insuficiencia orgánica múltiple $\mathrm{y}$, por ende, altas tasas de mortalidad. Antes de la era de los antibióticos la mortalidad por MND era superior al $50 \%{ }^{3}$. A pesar de los avances en antibióticos, el diagnóstico por imágenes y el manejo quirúrgico, la MND sigue siendo asociada actualmente a altas tasas de mortalidad que van desde el $11 \%$ al $40 \%$ aproximadamente ${ }^{7,8}$. El diagnóstico precoz, la cirugía de debridación temprana y la antibioterapia según cultivos, determinan la sobrevida de los pacientes ${ }^{9}$.

Este reporte tiene como objetivo presentar un caso de MND de origen odontogénico en un hombre de mediana edad, el cual se trató y controló con éxito.

\section{Caso clínico}

Paciente género masculino de 34 años de edad en situación de calle, sin antecedentes mórbidos, consulta en el Servicio de Urgencia Odontológica y Maxilofacial del Hospital de Urgencia Asistencia Pública, por presentar un aumento de volumen facial y cervical derecho de 3 días de evolución. El examen físico general reveló: Pulso $=85$ latidos por minuto; presión arterial $=118 / 83 \mathrm{mmHg}$; temperatura $=38{ }^{\circ} \mathrm{C}$; saturación de oxígeno $=97 \%$; respiración $=17$ respiraciones por minuto, con síntomas y signos asociados a compromiso del estado general como anorexia, astenia, adinamia, disfagia y odinofagia. Al examen físico segmentario de cabeza y cuello presentó un aumento de volumen submandibular y cervical derecho difuso con extensión al tórax, indurado, con cambio de coloración de la piel, doloroso a la palpación, aumento de temperatura local y limitación de la movilidad cervical (Figura 1). El examen bucal evidenció la presencia de trismus moderado, paladar blando eritemotoso, vestíbulo bucal inferior derecho ocupado en relación a diente 4.7 con caries profunda y diente 4.8 semierupcionado.

Inicialmente se realizó el ingreso hospitalario, se indicó antibioterapia (ceftriaxona/clindamicina) junto con analgesia endovenosa. Se solicitó una tomografía computarizada (TC) contrastada de cabeza, cuello y toráx para evaluar espacios anatómicos comprometidos y se indicó exámenes de laboratorio (perfil hematológico, glicemia, electrolitos plasmáticos, creatinina, proteína $\mathrm{C}$ reactiva [PCR], procalcitonina y perfil de coagulación).

El TC reveló como impresión diagnóstica un extenso compromiso inflamatorio-infeccioso cervical descendente hacia el toráx. Colecciones en espacio masticatorio y parafaríngeo. Signos sugerentes de fascitis necrotizante descente. Compromiso mediastínico y de la vía aérea (Figura 2). En los exámenes de laboratorio destacó una leucocitosis de 20,4 x $10^{3} \mathrm{~mm}^{3}[4,0-9,0]$, una PCR elevada de $50,8 \mathrm{mg} / \mathrm{dL}$ $[0-0,5]$ y una procalcitonina elevada de $23,43 \mathrm{ng} /$ dL $[0-0,5]$.

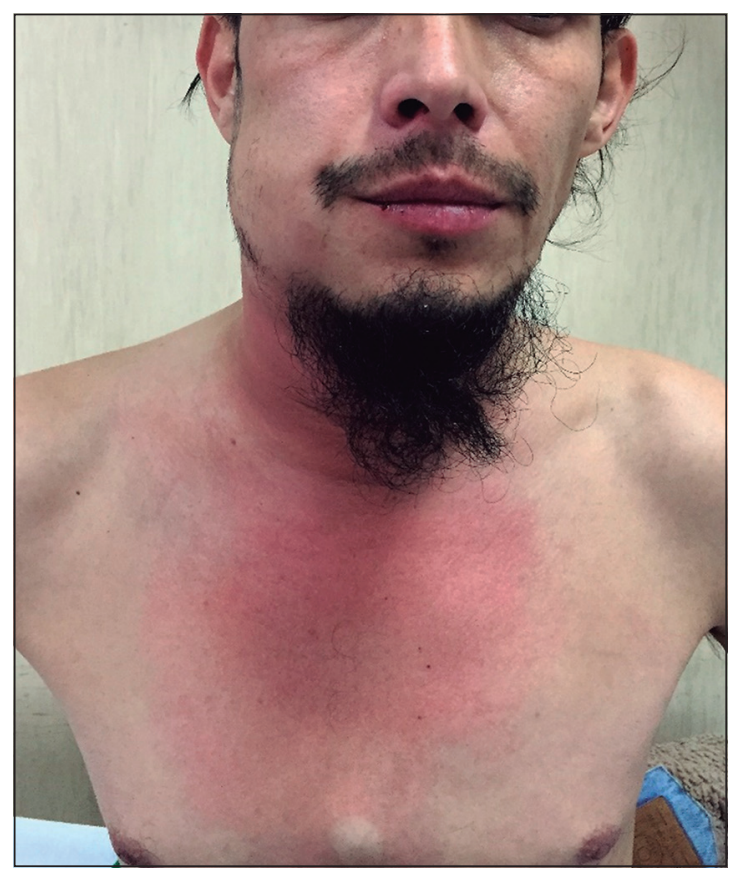

Figura 1. Examen físico segmentario de cabeza y cuello. Aumento de volumen submandibular y cervical derecho difuso con extensión al tórax, de consistencia indurada, piel enrojecida, doloroso a la palpación, aumento de temperatura local y limitación de la movilidad cervical. 


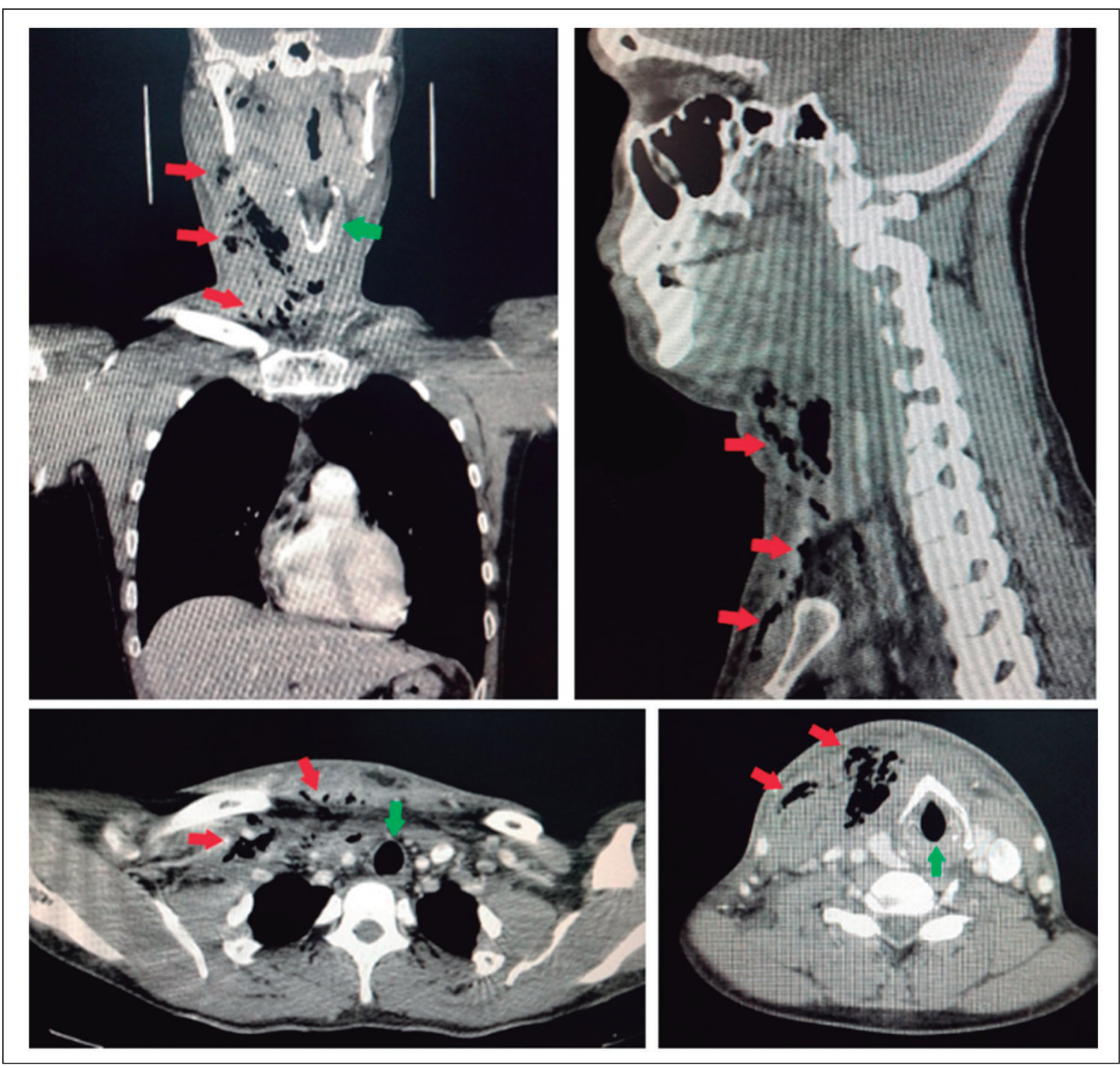

El plan de tratamiento se ejecutó bajo anestesia general. La técnica quirúrgica fue mediante una cervicotomía abordando vía pre-esternocleidomastoideo derecho, donde se identificó una importante colección purulenta en el espacio parafaríngeo que por cefálico comprometía el piso de la boca y por caudal comprometía, por anterior del manubrio del esternon y el mediastino antero-superior (Figura 3). Conjuntamente se realiza biopsia y se envía a cultivo las secreciones, debridamiento de espacios comprometidos y aseo prolijo con abundante solución fisiológica. Se instalaron drenajes de media caña en espacios parafaríngeo, submandibular, preesternal y mediastino antero-superior que se fijaron a la piel con sutura seda 3/0 (Figura 4). En un segundo tiempo quirúrgico, pero a continuación, se realizó la defocación de los dientes causales. Paciente queda hospitalizado en la Unidad de Cuidados Intensivos (UCI) con soporte médico, antibioterapia (ceftrixona/clindamicina) y analgesia endovenosa, además de exámenes de laboratorio diarios y TC de control para evaluar evolución del paciente. En cuanto a los exámenes de laboratorio se observó una buena evolución de los parámetros inflamatorios e infecciosos (recuento leucocitario, PCR y procalcitonina). En el TC de control se observaron drenajes bien posicionados, ubicados en espacios comprometidos y signos de remisión del proceso inflamatorio/infeccioso. El resultado del cultivo de secreciones determinó un desarrollo de Streptococcus anginosus, microorganismo susceptible a la antibioterapia endovenosa indicada. Posteriormente, se continúa con exámenes
Figura 2. Diferentes cortes de la TC a nivel de cabeza, cuello y torax, flechas rojas indican difusión del proceso infeccioso y flechas verdes compromiso de la vía aérea. 
Figura 3. Cervicotomía vía pre-esternocleidomastoideo derecho. Se observa importante contenido purulento asociado a tejido necrótico circundante.

Figura 4. Drenajes con tubos de silicona tipo media caña en espacios submandibular, parafaríngeo, preesternal y mediastino antero-superior dispuestos de cefálico a caudal respectivamente.
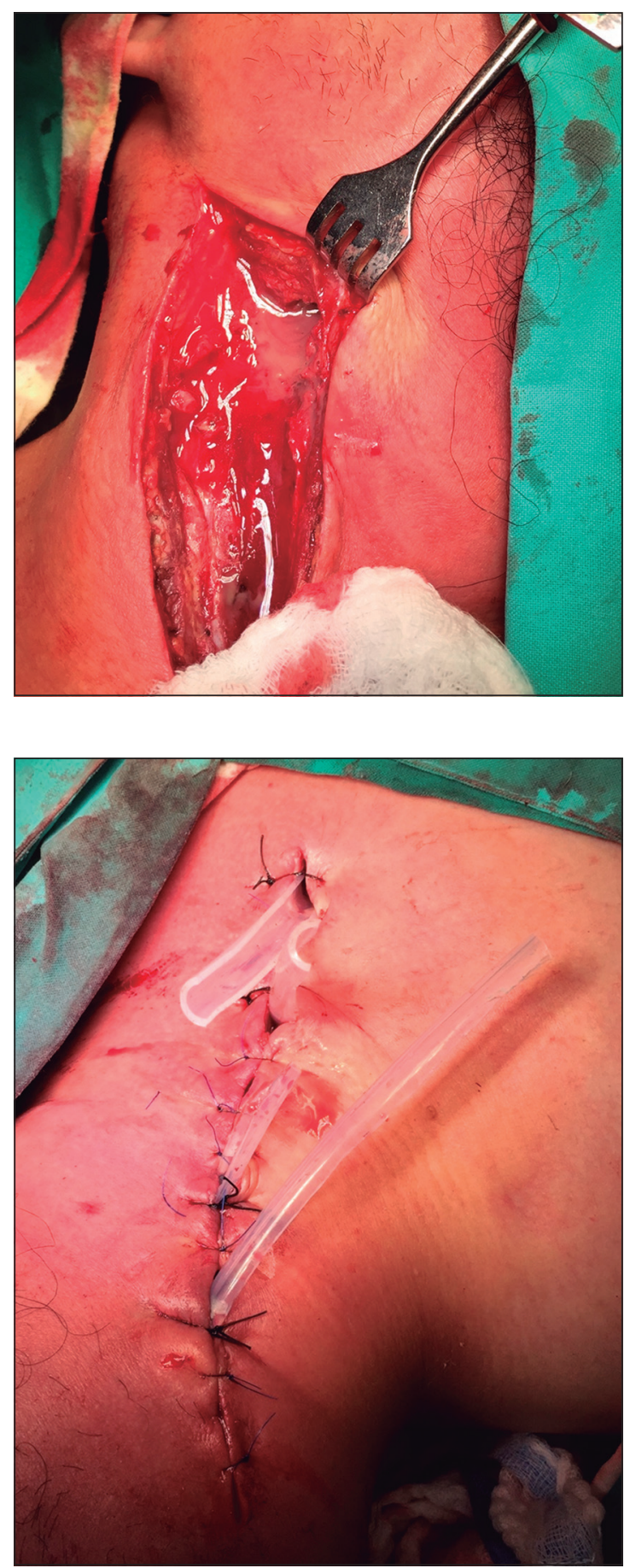

de laboratorio y controles imageneológicos junto con aseos quirúrgicos locales periódicos hasta lograr el alta del paciente a los 16 días. Finalmente, se realizan controles ambulatorios para el manejo de heridas quirúrgicas, evaluando función y evolución satisfactoria.

\section{Discusión}

Las infecciones odontogénicas son generalmente localizadas y se pueden tratar mediante terapias convencionales. A pesar de esto, si estas infecciones no pueden controlarse, ya sea por no realización de tratamientos oportunos o por estados inmunosuprimidos del paciente, se pueden desarrollar diferentes complicaciones como lo son bacteremias, endocarditis bacteriana, infecciones cervicales o incluso mediastinitis ${ }^{2,10}$.

Según Estrera et al., los criterios diagnósticos para la MND son: (a) síntomas/signos de una infección orofaríngea clínicamente grave; (b) hallazgos imageneológicos de mediastinitis mediante TC; (c) presencia de una infección mediastínica que se detecta durante la cirugía y (d) una relación entre una infección orofaríngea y una $\mathrm{MND}^{10}$. Teniendo en cuenta estos parámetros, en este caso se observó una infección en la región submandibular y cervical derecha con extensión al tórax, asociada a trismus, dolor, enrojecimiento y aumento de volumen de consistencia indurada. En el TC se identificó una obstrucción parcial de la vía aérea con estrechamiento hacia el lado contralateral de la infección, además de colecciones a nivel cervical en relación con el borde anterior del músculo esternocleidomastoideo y regiones vecinas a éste.

La TC contrastada de cuello y tórax se debe realizar lo antes posible en pacientes con infecciones cervicales o con sospecha de MND con el objetivo de determinar si hay extensión al mediastino, establecer el diagnóstico y ser el punto de comparación para el control posoperatorio ${ }^{11-13}$. En este caso, al momento de tener sospecha de MND, inmediatamente se realizó una TC contrastada en la que ayudó a definir el diagnóstico y el abordaje terapéutico. Además se realizaron $\mathrm{TC}$ controles que ayudaron a determinar la involución del proceso infeccioso.

En el presente caso la diseminación del absceso se clasificó como Endo Tipo 1, es decir, MND limitada a la parte superior del mediastino ${ }^{14}$, en el cual se logró una regresión de los síntomas después del drenaje y eliminación del tejido necrótico mediante un abordaje transcervical. Además, fue necesario realizar las exodoncias de los dientes causales.

El tratamiento médico y la terapia antibacteriana también es un componente importante en el tratamiento de la MND de origen odontogénico. La antibioterapia debe aplicarse durante un tiempo prolongado, usando antibióticos endovenosos de amplio espectro que cubran inicialmente estreptococos y bacterias anaeróbicas, que posteriormente se ajustará con los resultados del cultivo ${ }^{12}$. En este 
caso se administró la combinación de ceftriaxona más clindamicina endovenoso, con posterior uso de amoxicilina más ácido clavulánico vía oral.

Finalmente, es importante la integración de un equipo multidisciplinario que convoque todas las especialidades que ayuden a tratar esta patología, para así estandarizar un protocolo que permita un diagnóstico oportuno y un tratamiento quirúrgico eficaz ${ }^{10,15}$.

\section{Conclusión}

La MND se debe sospechar clínicamente y confirmarse inmediatamente con una TC. Un diagnóstico rápido, un tratamiento quirúrgico agresivo, más la terapia antibiótica adecuada y la atención de apoyo, son los pilares fundamentales para el manejo de esta patología. El tratante debe tener una actitud expectante frente a cualquier difusión de la infección y/o evolución desfavorable o no esperada del enfermo.

\section{Responsabilidades éticas}

Protección de personas y animales. Los autores declaran que para esta investigación no se han realizado experimentos en seres humanos ni en animales.

Confidencialidad de los datos. Los autores declaran que en este artículo no aparecen datos de pacientes.

Conflictos de interés: no hay.

\section{Bibliografía}

1. Pinto A, Scaglione M, Scuderi MG, Tortora G, Daniele S, Romano L. Infections of the neck leading to descending necrotizing mediastinitis: role of multi-detector row computed tomography. Eur J Radiol. 2008;65:38994. DOI: 10.1016/j.ejrad.2007.09.024.

2. Kocher GJ, Hoksch B, Caversaccio M, Wiegand J, Schmid RA. Diffuse descending necrotizing mediastinitis: surgical therapy and outcome in a singlecentre series. Eur J Cardiothorac Surg. 2012;42:e66-e72. DOI: 10.1093/ejcts/ ezs385.

3. Pearse HE. Mediastinitis following cervical suppuration. Annals of Surgery 1938;108:588-611. DOI: 10.1097/00000658-19381000000009

4. Freeman RK, Vallieres E, Verrier ED, Karmy-Jones R, Wood DE. Descending necrotizing mediastinitis: An analysis of the effects of serial surgical debridement on patient mortality. J Thorac Cardiovasc Surg. 2000;119:260-7. DOI: 10.1016/ S0022-5223(00)70181-4.

5. Li KK, Varvares MA, Meara JG. Descending necrotizing mediastinitis: a complication of dental implant surgery. Head \& neck. 1996; 18(2):192-6. DOI: 10.1002/(SICI)1097-
0347(199603/04)18:2<192::AID-

HED11 >3.0.CO;2-H.

6. González García R, Risco Rojas R, Román Romero L, Moreno-García C, López García C. Descending necrotizing mediastinitis following dental extraction. Radiological features and surgical treatment considerations. J Craniomaxillofac Surg. 2011;39:335-9. DOI: 10.1016/j.jcms.2010.10.001

7. Roccia F, Pecorari GC, Oliaro A, Passet E, Rossi P, Nadalin J, et al. Ten years of descending necrotizing mediastinitis: management of 23 cases. J Oral Maxillofac Surg. 2007;65:1716-24. DOI: 10.1016/j.joms.2006.10.060.

8. Ridder GJ, Maier W, Kinzer S, Teszler CB, Boedeker CC, Pfeiffer J. Descending necrotizing mediastinitis: contemporary trends in etiology, diagnosis, management, and outcome. Annals of Surgery 2010;251:528-34. DOI: 10.1097/ SLA.0b013e3181c1b0d1.

9. Corsten MJ, Shamji FM, Odell PF, Frederico JA, Laframboise GG, Reid KR, et al. Optimal treatment of descending necrotising mediastinitis. Thorax 1997;52:702-8. DOI: 10.1136/ thx.52.8.702

10. Estrera AS, Landay MJ, Grisham JM, Sinn DP, Platt MR. Descending necrotizing mediastinitis. Surg Gynecol Obstet. 1983;157:545-52.
11. Sakamoto H, Aoki T, Kise Y, Watanabe D, Sasaki J. Descending necrotizing mediastinitis due to odontogenic infections. Oral surgery Oral medicine Oral pathology. 2000;89:412-9. DOI: 10.1016/s1079-2104(00)70121-1.

12. Prado-Calleros H, Jiménez-Fuentes E, Jiménez-Escobar I. Descending necrotizing mediastinitis: systematic review on its treatment in the last 6 years, 75 years after its description. Head Neck. 2016;38 Suppl 1:E2275-83. DOI: $10.1002 /$ hed.24183.

13. Misthos P, Katsaragakis S, Kakaris S, Theodorou D, Skottis I. Descending necrotizing anterior mediastinitis: analysis of survival and surgical treatment modalities. J Oral Maxillofac Surg. 2007;65:635-9. DOI: 10.1016/j. joms.2006.06.287.

14. Endo S, Murayama F, Hasegawa T, Yamamoto S, Yamaguchi T, Sohara Y, et al. Guideline of surgical management based on diffusion of descending necrotizing mediastinitis. Jpn J Thorac Cardiovasc Surg. 1999;47:14-9. DOI: $10.1007 / \mathrm{bf0} 3217934$.

15. Soylu E, Erdil A, Sapmaz E, Somuk BT, Akbulut N. Mediastinitis as complication of odontogenic infection: A case report. Niger J Clin Pract. 2019;22:869-71. DOI: 10.4103/njcp. njcp_539_18. 